Meta

Journal des traducteurs

Translators' Journal

\title{
Le verbum interius du traducteur et la cristallisation du sens : la traçabilité du processus traduisant à travers les isotopies et les sciences cognitives
}

\section{Bernd Stefanink et Ioana Bălăcescu}

Volume 62, numéro 2, août 2017

URI : https://id.erudit.org/iderudit/1041025ar

DOI : https://doi.org/10.7202/1041025ar

Aller au sommaire du numéro

\section{Éditeur(s)}

Les Presses de l’Université de Montréal

\section{ISSN}

0026-0452 (imprimé)

1492-1421 (numérique)

\section{Découvrir la revue}

\section{Citer cet article}

Stefanink, B. \& Bălăcescu, I. (2017). Le verbum interius du traducteur et la cristallisation du sens : la traçabilité du processus traduisant à travers les isotopies et les sciences cognitives. Meta, 62(2), 289-312.

https://doi.org/10.7202/1041025ar

\section{Résumé de l'article}

Nous nous sommes proposé dans ce travail de mettre en évidence le bien-fondé de certaines thèses qui sont à la base de l'approche herméneutique en traduction, mais ont donné lieu à des critiques de la part d'un certain nombre de traductologues qui, selon nous, font preuve d'une méconnaissance de cette approche. Dans la conclusion de la première synthèse des recherches en herméneutique traductive, Cercel (2013: 364) déplore ce manque de compréhension dû, selon elle, à un manque d'explicitation de ces thèses de la part de ses représentants, qui s'en tiennent à des "plakative Aussagen » [déclarations abstraites]. Nous relevons ce défi d'explicitation en essayant d'établir le bien-fondé de ces thèses contestées, notamment celle que résume cette déclaration de Heidegger " Den Bedeutungen wachsen Worte zu » [les mots viennent aux significations] - et en illustrant des concepts comme la Leibhaftigkeit (Paepcke 1986) [corporéité] à l'aide d'un exemple pratique tiré de notre corpus d'herméneutique traductive. Nous utilisons l'analyse conversationnelle ethnométhodologique pour étudier la négociation du sens à traduire en ayant pour objectif de fournir des explications aux processus relationnels entre texte et traducteur. Nous nous basons, pour ce faire, sur des théories linguistiques (notamment le concept d'isotopie), ainsi que sur des recherches récentes en sciences cognitives. Notre exemple veut illustrer la thèse que le sens élaboré par le traducteur dans son mental se comporte comme le verbum interius tel que le conçoit Saint-Augustin.
Ce document est protégé par la loi sur le droit d'auteur. L’utilisation des services d'Érudit (y compris la reproduction) est assujettie à sa politique d'utilisation que vous pouvez consulter en ligne.

https://apropos.erudit.org/fr/usagers/politique-dutilisation/ 


\title{
Le verbum interius du traducteur et la cristallisation du sens: la traçabilité du processus traduisant à travers les isotopies et les sciences cognitives
}

\author{
BERND STEFANINK \\ Université de Bielefeld, Allemagne \\ Universidade Federal de Santa Catarina, Brésil \\ bstefanink@hotmail.com
}

IOANA BĂLĂCESCU

Université de Craiova, Roumanie

ioanadi@hotmail.com

\begin{abstract}
RÉSUMÉ
Nous nous sommes proposé dans ce travail de mettre en évidence le bien-fondé de certaines thèses qui sont à la base de l'approche herméneutique en traduction, mais ont donné lieu à des critiques de la part d'un certain nombre de traductologues qui, selon nous, font preuve d'une méconnaissance de cette approche. Dans la conclusion de la première synthèse des recherches en herméneutique traductive, Cercel (2013: 364) déplore ce manque de compréhension dû, selon elle, à un manque d'explicitation de ces thèses de la part de ses représentants, qui s'en tiennent à des «plakative Aussagen» [déclarations abstraites]. Nous relevons ce défi d'explicitation en essayant d'établir le bien-fondé de ces thèses contestées, notamment celle que résume cette déclaration de Heidegger «Den Bedeutungen wachsen Worte zu» [les mots viennent aux significations] - et en illustrant des concepts comme la Leibhaftigkeit (Paepcke 1986) [corporéité] à l'aide d'un exemple pratique tiré de notre corpus d'herméneutique traductive. Nous utilisons l'analyse conversationnelle ethnométhodologique pour étudier la négociation du sens à traduire en ayant pour objectif de fournir des explications aux processus relationnels entre texte et traducteur. Nous nous basons, pour ce faire, sur des théories linguistiques (notamment le concept d'isotopie), ainsi que sur des recherches récentes en sciences cognitives. Notre exemple veut illustrer la thèse que le sens élaboré par le traducteur dans son mental se comporte comme le verbum interius tel que le conçoit Saint-Augustin.
\end{abstract}

\section{ABSTRACT}

Our aim is to show the validity of a certain number of theses which are fundamental in the hermeneutic approach of translation, but which have led to criticism from a number of translatologists, due to their lack of familiarization with the hermeneutical approach. In the conclusion of the first summa which provides a state of the art in translational hermeneutics, Cercel (2013: 354) deplores this lack of understanding and attributes it to a lack of expliciteness which caracterizes the abstract statements [plakative Aussagen] of the representatives of the hermeneutical approach in translation. We meet this challenge by trying to establish the validity of the contested theses - including this statement of Heidegger «Den Bedeutungen wachsen Worte zu» [Words grow into the meanings] - and by illustrating concepts such as the Leibhaftigkeit (Paepcke 1986) [corporeality] of the translator, using a practical example from our corpus of translational hermeneutics. Our method: we use conversational ethnomethodological analysis to study the negotiation 
of the meaning to be translated. Our aim is to explain the processes that manage the relationship between text and translator based on linguistic theories (in particular, the concept of isotopy) as well as on recent research in the field of cognitive sciences. Our thesis: The meaning that the translator constructs in his brains is the verbum interius as it is conceived by Augustinus.

\section{RESUMEN}

En este trabajo nos hemos propuesto poner en evidencia la razón de ser de algunas tesis en las que se basa el enfoque hermeneútico en traducción, pero que dieron lugar a críticas por parte de algunos traductólogos que, en nuestra opinión, no conocen bien este enfoque. En la conclusión de la primera síntesis de las investigaciones en hermeneútica traductiva, Cercel (2013:364) lamenta esta falta de comprensión debida, según ella, a una explicitación insuficiente de estas tesis por sus representantes quienes se limitan a plakative Aussagen [declaraciones abstractas]. Asumimos el desafío de explicitar estableciendo la razón de ser de estas tesis cuestionadas, en particular la resumida por la declaración de Heidegger «Den Bedeutungen wachsen Worte zu» [las palabras alcanzan las significaciones] - y utilizando conceptos como Leibhaftigkeit (Paepcke 1986) [corporeidad,»] del traductor mediante un ejemplo práctico de nuestro corpus de hermeneútica traductiva. Utilizamos el análisis conversacional etnometodológico para estudiar la negociación del sentido por traducir con la finalidad de dar explicaciones acerca de los procesos relacionales entre texto y traductor. Para ello, nos basamos en teoría lingüísticas (en especial el concepto de isotopía) así como en investigaciones recientes en ciencias cognitivas. Nuestro ejemplo apunta a ilustrar la tesis según la cual el sentido eleborado por el traductor en su mente se comporta como el verbum interius tal como lo concibe San Agustín.

\section{MOTS CLES/ KEYWORDS/PALABRAS CLAVE}

herméneutique traductive, analyse conversationnelle ethnométhodologique, sciences cognitives, créativité, isotopie

translational hermeneutics, ethnomethodological conversation analysis, translation and cognitive research, creativity, isotopy

hermeneútica traductiva, análisis conversacional etnometodológico, ciencias cognitivas, creatividad, isotopía

\section{Préliminaires: nos motivations}

Cet article doit son élaboration à plusieurs nécessités convergentes:

- les étudiants que nous encadrons dans le cadre de notre mission au Brésil ont souvent comme sujets de thèses de doctorat la traduction commentée d'un texte littéraire; or, l'approche herméneutique, qui leur serait pourtant de la plus haute utilité, est peu connue;

- les critiques formulées par certains traductologues à l'égard de l'approche herméneutique nous semblent témoigner d'une méconnaissance de cette approche;

- le bilan dressé par Cercel (2013: 364), qui attribue cette même méconnaissance aux formulations trop abstraites des représentants de cette approche, nous paraît être un défi justifiant une tentative d'entrer dans le détail de ce que les philosophes herméneutes ont pu entendre par un sens qui est derrière les mots selon Gadamer ou «entre les lignes du texte» (Schleiermacher 1977:315) ou encore dans ce que Ricoeur nomme «l'orient du texte» (1975: 156).

Pour ce qui est du troisième point, une des formulations quelque peu abstraites dont parle Cercel, qui dans leur abstraction peuvent laisser perplexe, nous vient de 
Heidegger (1927/1967'11 160$)$ : «Den Bedeutungen wachsen Worte zu" [les mots viennent aux significations] $]^{1}$, qui a été souvent mal traduite parce que les traducteurs ont négligé de mettre en évidence l’antériorité de la pensée par rapport aux mots. Notre travail veut illustrer non seulement cette antériorité, mais aussi le caractère graduel qu'implique le wachsen [croître] de cette croissance des mots vers les significations.

Pour ce qui est du deuxième point qui nous a conduits à relever le défi lancé par Cercel, nous nous proposons de montrer que les critiques de certains traductologues sont peu fondées et relèvent d'une méconnaissance de l'approche herméneutique.

Par exemple, nous espérons détromper Gerzymisch (2013: 71) quant au manque de transparence de l'approche herméneutique. Pour Gerzymisch cette transparence est liée, entre autres, à la recherche de mots concrets qu'on peut "'trans'-porter» (Gerzymisch 2013: 74) dans la langue cible. Gerzymisch prétend que le «désespoir» de Lenz, héros de la nouvelle du même nom de Büchner, ne peut se traduire que si l'on trouve une «expression concrète» (Gerzymisch 2013: 73) qui le représente dans le texte source: "C'est seulement cette expression que nous pouvons "'trans'-porter» ['über'-setzen] (Gerzymisch 2013: 74) et «non pas le sens global qu'on a dégagé (par exemple l'état d'anxiété dans lequel se trouve Lenz)» (Gerzymisch 2013: 73). Cette affirmation touche à un point vital de l'approche herméneutique qui, au contraire, se veut holistique.

Face à l'approche herméneutique, Gerzymisch concède que le traducteur peut, certes, dégager le sens intuitivement, mais que ce processus ne peut alors être décrit. Selon elle, c'est cette «transparence interindividuelle» qui manquerait à l'approche herméneutique ${ }^{2}$.

Nous nous proposons de montrer primo que c'est bien le sens global qui s'impose dans la négociation traductive que nous allons présenter et secundo que l'examen de cette négociation, après analyse, satisfait pleinement à l'exigence de transparence qui conditionne le caractère scientifique d'une approche.

Quant à la réception par Christine Durieux de la théorie interprétative, dont elle critique la notion de déverbalisation, elle est fondée sur une mauvaise compréhension d'un aspect fondamental de l'herméneutique:

Toutefois, cette approche herméneutique de la saisie du sens remet en cause la notion de déverbalisation. De fait, qu'est-ce qu'une pensée nue sans support verbal?

L'affirmation de l'existence d'une phase de déverbalisation s'intercalant entre compréhension et réexpression n'est guère tenable, le sens déverbalisé flottant entre deux langues est un peu comme on peut être assis entre deux chaises (Durieux 2009: 354).

Rectifions et précisons: l'herméneutique n'a jamais parlé d'un sens flottant entre deux langues. Par contre, l'herméneutique philosophique a pu prétendre que le sens se trouvait «entre les lignes» (Schleiermacher 1977: 315), formulation à laquelle nous préférons celle qui est nous est suggérée par l'analyse de notre corpus: le sens se trouve entre les isotopies du texte et se structure sous le regard du lecteur.

\section{Les fondements théoriques de notre expérience}

\subsection{Le 'verbum interius' à la base de l'approche herméneutique}

Dans son Einführung in die Hermeneutik, Grondin décrit une rencontre avec Gadamer dans une de ces chaleureuses Wirtsstuben de la ville des philosophes qu'est 
Heidelberg. Grondin posait le problème de l'universalité de l'herméneutique: en quoi cette universalité consistait-elle? Après tout ce qu'il avait lu sur le sujet, Grondin s'attendait à une réponse longue et quelque peu vague. Après un instant de réflexion Gadamer répondit en deux mots: «Dans le verbum interius» (Grondin 1991/2012: 9). Ajoutant, quelques minutes plus tard, que cela lui venait de Saint-Augustin, qui explique que l'on ne peut jamais exprimer tout ce qu'on renferme en son for intérieur et qu'il y a toujours en nous une lutte inlassable [ein Ringen um Worte] pour trouver «les mots pour le dire».

Il semble pourtant qu'au moment de cette rencontre, en automne 1988, Gadamer n'ait pas encore totalement pris conscience de la portée de cette révélation ${ }^{3}$ et c'est à juste titre que Grondin (1991: 9) nous décrit son étonnement: cette affirmation n'allait-elle pas à l'encontre de la thèse fondamentale de la philosophie gadamérienne aux termes de laquelle tout est dans le langage? Quant au terme même de verbum interius, s'il fait effectivement son apparition dans Vérité et Méthode, celle-ci n'est que périphérique, loin de l'importance qu'elle prendra dans le discours ultérieur de Gadamer. La référence à Saint-Augustin (rapportée par Grondin (1991 : 12) est significative, car si les philosophes qui se sont occupés des rapports entre pensée/sens et langage ont toujours conclu à un verbe intérieur qu'il s'appelle logos endiathetos chez les stoïciens, verbum mentis chez Aquinas, ou encore actus exercitus ${ }^{4}$, celui-ci était, comme dans le Cratyle de Platon, considéré comme une idée statique dont la représentation linguistique n'était qu'une copie, le langage n'ayant qu'une fonction ancillaire. Dans la tradition chrétienne, par contre, telle que la représente Saint-Augustin, ce concept est lié à la Trinité où Dieu est le Verbe, mais ce Dieu-Verbe s'incarne aussi dans la personne du Fils de Dieu comme nous le révèle l'Évangile selon Saint-Jean. Or, dans la tradition chrétienne, le Fils de Dieu est censé permettre aux humains de mieux comprendre Dieu, dans la mesure où il est un Dieu à visage humain. Transféré sur la relation entre pensée/sens et langage, le langage acquiert une nouvelle importance dans une dynamique entre pensée/sens et expression linguistique du sens: il permet de mieux comprendre la pensée. De même que les rapports entre Dieu et son incarnation comme fils de Dieu sont très étroits - ils sont un -, de même il n'y a pas une séparation nette entre d'un côté un verbe intérieur qui serait une pensée finie (comme chez Platon) et sa représentation linguistique. Et Gadamer de se référer au texte de Kleist ${ }^{5}$ (1805) Über die allmähliche Verfertigung der Gedanken beim Reden [Sur l'élaboration progressive des idées par la parole], dans lequel Kleist relate comment des idées, encore vagues et embrouillées dans sa tête, arrivent à se préciser et prendre forme lorsqu'il commence à les énoncer et finissent par devenir claires sous la pression de devoir terminer ses phrases. Il faut imaginer un va-et-vient entre langage et pensée dans lequel le langage a un rôle dans la construction du sens.

N'avons-nous pas là une illustration de la relation entre sens à traduire et expression en langue cible caractéristique du processus traduisant? Kleist nous dit bien que c'est en parlant à sa sœur, qui n'est absolument pas en mesure de suivre son discours juridique ou littéraire, que ce processus d'éclaircissement de sa pensée par la parole se produit, par le simple fait de devoir communiquer ce qu'il a dans sa tête. N'est-ce pas là la situation dans laquelle se trouve le traducteur, obligé de formuler en langue cible un sens construit à partir du texte source, et qui se clarifie en évoluant et prend forme devant cette obligation de formuler? Comme nous l'avons démontré dans Bălăcescu et Stefanink (2012), la Stimmigkeit de la traduction ne sera parvenue à son 
aboutissement qu'avec le sentiment que le sens qui s'est construit dans le mental du traducteur - pas une fois pour toutes, mais graduellement au cours du processus de rédaction en langue cible, en passant par des étapes de solutions traduisantes tentatives - se retrouve bien dans le texte traduit. Le «Vorverständnis » [pré-compréhension] heideggerien du texte (à traduire) qui selon Gadamer initie le cercle herméneutique de la compréhension, n'arrivera à son assouvissement, à sa complétion ${ }^{6}$ et à son harmonie avec le texte traduit qu'à la fin de ce processus.

C'est exactement le processus que l'on observe chez nos informateurs au cours de l'expérience traduisante que nous allons présenter dans ce qui suit: leur verbum interius est la représentation mentale du sens qu'ils se sont construit à partir du texte source, représentation du sens pour laquelle ils tentent de trouver les mots en langue cible.

On nous signale qu'une référence bibliographique utile à ce sujet est Mirela Oliva (2009), Das innere Verbum in Gadamers Hermeneutik, Tübingen: Mohr Siebeck. Nous n'avons malheureusement pas pu y avoir accès.

\subsection{La «Leibhaftigkeit»ou «somatique»du traducteur}

Dans son ouvrage sur Langage et émotion, Schwarz-Friesel (2007) a, contrairement à ce qu'elle avait prôné dans ses ouvrages antérieurs, démontré la priorité de la perception sensuelle et émotionnelle sur la saisie logique, plaidant ainsi pour une intégration des émotions dans le champ des études cognitivistes. Ce faisant, elle tient compte des recherches du neurologue Damasio (1994) qui a démontré, en examinant des lésions du cerveau, que raison et émotion sont «intimement liées» (1994: 74) dans le cortex préfrontal et que le manque émotionnel dû aux lésions cérébrales nuit à des prises de décision logiques. Avant Damasio, Robinson (1991: 34) conseillait déjà au traducteur de se fier à son propre "somatic sense of appropriateness», de «sentir» ce qu'il traduisait.

C'est là le comportement traduisant que notre corpus herméneutique illustre de façon convaincante. En entrant dans un dialogue (gadamérien) avec un texte qui fait, de façon privilégiée, appel aux sens, nos informateurs «se laissent prendre au jeu» comme le préconise Gadamer, entrent dans la peau du texte et, «imprégnés » ${ }^{7}$ par celui-ci, produisent en langue cible un reflet de ces impressions. Fritz Paepcke a introduit pour cela le terme de Leibhaftigkeit [corporéité] (1981: 125), que Stolze (2011a: 67) a rendu, à l'écoute des cognitivistes anglo-saxons, par embodiment auquel nous préférons cependant le terme plus motivé de corporeality, introduit par Robinson (1991: 94). Nous y reviendrons plus bas, après avoir analysé notre corpus conversationnel. Avec ce terme de Leibhaftigkeit, Paepcke a voulu insister sur le fait que le traducteur est impliqué, non seulement avec son intellect, mais avec tout son être dans le processus de traduction, avec ses sens, ses sentiments, ses expériences, soit le traducteur en tant qu'être social et émotionnel.

\section{Fondements méthodologiques : analyse conversationnelle ethnométhodologique et dialogue gadamérien}

La méthode d'investigation dont nous nous servons ${ }^{8}$ a été qualifiée de «intrinsèquement herméneutique» (Cercel 2013: 143). Elle repose, d'une part, sur l'idée gadamérienne d'un dialogue avec le texte, assorti, en outre, d'un dialogue entre les participants 
qui doivent négocier le sens de ce texte 9 . D’autre part cette méthode est tributaire de l'analyse conversationnelle ethnométhodologique, telle qu'elle a été développée par les sociologues américains dans les années soixante-dix du siècle dernier, afin d'étudier les idées naïves des gens, telles qu'elles apparaissent dans leur discours au quotidien, notamment à propos d'un domaine de spécialité scientifique (Garfinkel 1984). Il y a ainsi, par exemple, l'ethnomédecine, qui étudie les métaphores que l'homme de la rue associe avec les différents acteurs qui interviennent dans les professions médicales. De là notre idée d'une ethnotraductologie (Stefanink 1995) et celle d'une étude plus générale des Métaphores avec lesquelles nous vivons (Lakoff et Johnson 1980). L'analyse conversationnelle ethnométhodologique fournit une base empirique, parfaitement adaptée aux principes d'« ouverture au texte» (Stolze 2015: 90-92) préconisés par l'herméneutique. Le caractère ouvert et imprévisible de l'analyse conversationnelle ethnométhodologique, associé à l'esprit d'ouverture, qui est une des caractéristiques fondamentales de l'herméneutique, fournit une base méthodologique parfaitement adaptée à notre recherche.

\section{Les bases matérielles de l'expérience: informateurs, texte et corpus conversationnel}

\subsection{Les informateurs}

Il s'agit d'un groupe de quatre étudiants d'anglais de l'Université de Bucarest, que nous avons placés devant un texte anglais à traduire en roumain, avec la charge de négocier une version commune en langue cible. Cette négociation a été enregistrée et transcrite. Les étudiants ont été priés de lire le texte individuellement avant d'entrer dans le dialogue négociateur.

\subsection{Le texte à traduire}

Il nous a paru particulièrement intéressant de reprendre un texte déjà utilisé par Kußmaul (2000) afin de comparer les résultats de ses recherches sur la créativité avec ceux que pourrait apporter une approche herméneutique. Il s'agit d'un texte qui avait été publié dans le journal Newsweek le 28 février 1994 et qui décrit le comportement des Allemands de l'Est après la réunification avec l'Allemagne de l'Ouest en octobre 1989:

Sleek new cars speed along straightened and repaved autobahns. Shiny service stations come equipped with well-stocked convenience stores and gleaming self-service restaurants. Enormous supermarkets, furniture stores and shopping emporiums dot the east German landscape, and giant cranes stand tall against the sky. Every seat is filled at Dresden's magnificent neo-classical opera house: comfortable burghers sip French champagne during the intermissions. Even in grimy Bitterfeld, a mining and chemicals centre notorious for its pollution, well-dressed women from a nearby retirement home gather for creamy coffee and gigantic pastries at a Swiss-owned coffee shop. (Newsweek, 28 février 1994)

\subsection{Extrait du corpus conversationnel}

Voir Annexe. 


\section{Analyse du corpus conversationnel}

1) Şmecher (16)

Smecher a un double sens. Dans ce contexte initial, il peut être compris comme un adjectif qualifiant cuvânt [mot] et attire l'attention sur la nature traîtresse de ce mot. Nos informateurs situent d'emblée le mot comme étant un mot dont il faut se méfier. Mais dans la bouche de l'informateur 3 il peut aussi préfigurer le șecher de la ligne 44, où mașini șmechere désigne des voitures impressionnantes, des voitures qui en imposent.

\section{2) Elegante (16)}

Le premier correspondant qui leur vient à l'esprit est elegante. C'est la traduction qu'on trouve dans les dictionnaires lorsque le mot se trouve dans un contexte de design ou d'automobiles. À titre de comparaison, la traduction automatique de Google pour sleek new cars en roumain est maşini elegante noi [des voitures neuves élégantes].

Cette solution pourrait paraître satisfaisante. Alors, pourquoi nos informateurs ne s'en satisfont-ils pas? Pourquoi trouvent-ils le mot sleek traître et pourquoi notre informateur 3 demande-t-il Vouă cum vi se pare? Nu e mai mult decât elegant? [Qu'en pensez-vous? N’est-ce pas bien plus qu'élégant?] (16-17), insécurisant l'informateur 1 qui répond: «je ne sais pas ...mais c'est un terme formel» (17-18).

\section{3) Strălucitoare (18) et lucios (19)}

Ce que l'informateur 1 veut dire par formel nous est révélé par l'informateur 2, qui ressent elegante comme manquant d'expressivité et propose strălucitoare (18) et lucios (19) deux synonymes pour brillant; ici lucios vient renforcer strălucitoare, dans d'autres contextes il pourrait aussi se traduire par reluisant si l'on veut marquer la différence par rapport à strălucitoare. Apparemment ces termes, plus expressifs, lui semblent plus adaptés pour décrire les voitures dans ce contexte.

4) Moderne (22), confortabile (23), scumpe (24)

Ceci incite l'informateur 3 à retourner au mot anglais sleek et à revenir à son idée que le mot «sleek» est sémantiquement «plus » que la traduction roumaine par elegante, il rajoute donc de nouveaux qualificatifs comme moderne (22), ce qui amène l'informateur 2 à rajouter à son tour confortabile (23), ce qui déclenche, par association, chez l'informateur 3 l'adjectif scumpe [cher] (24).

5) Extravagante (26) confortabile (23), confortabil (28)

Le flow, qui emporte un informateur qui - pour parler selon Gadamer (1960: 170) - s'est «laissé prendre au jeu» peut conduire à des exagérations, phénomène que Stendhal (1822), dans De l'amour, décrit comme un processus de cristallisation, dans lequel l'homme, dans un élan émotionnel, attribue à l'être aimé des qualités que celui-ci ne possède pas. Nous emprunterons ce terme à Stendhal, sans la valeur négative toutefois que Stendhal lui prête, mais en tant que terme scientifique désignant la concrétisation, en un mot ou en une expression, de ce sens qui flotte non pas, comme 
suppose Christine Durieux, entre les langues, mais entre les isotopies du texte, et nous verrons que même la proposition d'extravagante doit son origine à des éléments bottom up dans le texte: Nihil ex nihilo.

Si l'idée de traduire sleek par le mot roumain extravagante peut surprendre, l'argumentation avec laquelle l'informateur 1 rejette cette proposition n'est pas moins surprenante: extravagante ne récupérerait pas cette idée de confort nu recuperează idea asta de confort (26-27) qu'il pense avoir découvert dans le mot sleek, pour lequel il propose à un moment la traduction confortabile (22-23). Il y a pour lui un problème de compatibilité. Comme nous verrons plus bas il y a cette idée de confort dans le texte, mais elle ne fait pas partie des traits pertinents que l'informateur 1 croit devoir récupérer du mot sleek.

6) Văcsuite (28)

Après avoir écarté extravagante comme étant prea mult [trop] (25), l'informateur 3 revient sur l'idée de quelque chose de brillant en suggérant văcsuite [ciré, laqué] (28) qui évoque quelque chose de poli avec de la cire. Nous avons là encore une fois quelque chose d'extravagant et de créatif, même du point de vue lexical, parce qu'il s'agit d'un mot utilisé uniquement dans certains contextes précis. Il ne figure pas dans les dictionnaires bilingues courants, et le DEX (Dicționarul Explicativ al limbii Române, 2009) donne la définition uns cu vacs [rendu brillant avec de la cire] (vacs venant de l'allemand Wachs [cire]). Le caractère 'extravagant' de ce mot se voit confirmé par le fait qu'immédiatement après l'avoir prononcé l'informateur 3 se rétracte en quelque sorte, demandant: Se zice în română văcsuite? [ça se dit en roumain văcsuite?] (29) et d'entamer une discussion dans laquelle l'informateur 1, cherchant dans sa mémoire répond que cela se dit [se zice], qu'il a déjà entendu ce mot [eu am auzit] (30). Nous assistons dès lors, dans les propos de l'informateur 1, à une série de ce qu'on pourrait appeler des indicateurs scéniques, c'est-à-dire des éléments bottom up dans le texte, qui font référence à des éléments top down et indiquent qu'il pense à une scène qu'il a vécue. On peut observer comment ces indicateurs scéniques deviennent de plus en plus personnels dans la formulation, partant du mot văcsuite et mă duce cu gândul, [littéralement : me porte avec la pensée vers] (30) pour aboutir à amintesc [je me souviens] (33). Ceci souligne bien le caractère subjectif de nos perceptions (!), amintesc étant un indicateur scénique fort, c'est-à-dire explicite.

\section{7) Ultraspălate şi ultraaranjate (32)}

Ce qui favorise l'enchaînement associatif qui aboutit à la création de ces deux lexèmes, c'est un élément sémantique introduit par văcsuite. Văcsuite est le participe passé du verbe a văcsui [polir à l'aide de cire, cirer], qui fait intervenir un personnage qui exécute l'acte de cirer. Même au participe passé cet acteur est sous-jacent à la sémantique de ce mot, introduisant un aspect dynamique que les propositions antérieures, comme elegante, strălucitoare, lucios, moderne, confortabile, scumpe n'impliquaient en aucun cas. Cet aspect dynamique se retrouve dans les propositions ultraspălate, ultraaranjate, favorisant l'association entre ces trois qualificatifs et impliquant la vision d'un propriétaire de voiture en train d'astiquer sa nouvelle voiture en y mettant tout son soin. Il y a dans ce texte une isotopie du dynamisme et d'activité fébrile contagieuse, les mots se contaminant les uns les autres. 
Pour qui connaît la mentalité de l'ouvrier allemand, qu'il soit Allemand de l'Est récupéré après la réunification ou Spätaussiedler [réfugiés tardifs] des années cinquante, l'ouvrier allemand de l'Est, réfugié en Allemagne de l'Ouest a toujours mis sa fierté première à prendre femme (s'il était venu sans cet «attribut»), s'acheter une voiture et construire une maison: c'était le signe de la réussite sociale et de l'intégration. Il semble que l'auteur de cet article ait très bien réussi à communiquer cette impression. Nos traducteurs l'ont saisie et tenté de cristalliser cette atmosphère en des mots expressifs, la chaîne associative fonctionnant sur la base de l'élément commun : activité implicite, acteur que l'on sent derrière l'action ${ }^{10}$ selon le principe des Memory Organization Packets (MOPs) de Schank (1982) (Bălăcescu et Stefanink: 2003b).

\section{8) Ghetele văcsuite (35)}

Voyons comment continue le chemin vers une solution créative. Selon Fillmore (1976), les mots du texte sont des frames linguistiques qui déclenchent des scenes dans la mémoire du récepteur. Ces visualisations de scenes sont plus ou moins subjectives selon le vécu de celui-ci. Pour l'informateur 1 le mot văcsuite est automatiquement associé au mot ghetele [bottes]: il se rappelle son service militaire - cazone (34) pendant lequel il était obligé de cirer chaque jour ses bottes pour les garder reluisantes, ce qui déclenche dans sa mémoire une conotație ușor negativă [une connotation légèrement négative] (33). L'élément proéminent dans cette scène - le figure selon Ronald Langacker (1987) - ce sont les ghetele văcsuite [bottes cirées] (35).

\section{9) Ca scoase din cutie (42)}

Le locuteur 3 par contre, a une visualisation toute positive. C'est lui qui est le premier à dire que sleek est bien plus qu'elegant (18) et qui sera le plus actif à rechercher une solution qui s'éloigne de la signification de base et qui va dans le sens de l'opulence qu'il ressent dans le texte, comme: moderne (32), scumpe (24), văcsuite (29), ultraspălate și ultraaranjate (32).

C'est lui aussi qui est, depuis le début, continuellement à la recherche d'un mot qui récupère tout (25), un mot qui exprime un peu de tout (36). Et ce tout n'a rien à voir avec les traits pertinents du mot à traduire, mais relève des impressions que lui a laissé la lecture de l'ensemble du texte. Apparemment il visualise les bottes cirées dans une boîte à cadeaux, ce qui l'amène à proposer ca scoase din cutie [comme sorti de la boîte à cadeaux]. Évidemment sous l'influence sémantique du mot new qui le jouxte et l'assimile sémantiquement.

\section{6. Évaluation de cette solution créative}

Pour Ionescu (2004), la traduction de la première phrase d'un roman pouvait avoir une fonction préfiguratrice de la pensée et du style de l'auteur (Stefanink 2011). Pour nos informateurs le mot sleek semble exercer cette même fonction. Leur solution est d'autant plus convaincante qu'elle est trouvée malgré eux, dans l'innocence d'une approche holistique du texte, en dépit de leurs tentatives répétées de rendre les traits pertinents du mot sleek conformément à leurs maximes de traduction. Si ceci s'est produit malgré eux, c'est qu'ils ont été überwältigt [subjugués] par le Textgeschehen [la vérité (dynamique) du texte] (Stolze 2015). Imprégnés (Lenk 2014) par la vérité du texte, ils ont cherché inconsciemment, dans un moment de fusion avec l'horizon du 
texte, à projeter dans ce mot préfigurateur, toutes les émotions ressenties dans leur lecture holistique du texte.

Le résultat est une tournure idiomatique sous forme de métaphore lexicalisée, dont les trois informateurs qui sont entrés dans le jeu - conformément à l'approche du sens dans un dialogue avec le texte au sens gadamérien du terme - trouvent qu'elle a le mérite de rendre un peu de tout avec les nuances. En outre, elle est en conformité avec les Metaphors we live by (Lakoff et Johnson 1980) dans la culture cible en Roumanie. Il faut savoir que cette chaîne associative est soutenue par le contexte culturel sous forme de bagage cognitif [world knowledge]. Ca scoase din cutie est en effet une métaphore courante, lexicalisée dans le contexte culturel roumain, quand il s'agit, par exemple, de louer l'apparition et notamment la tenue vestimentaire, de vedettes du spectacle qu'on présente sur une scène; ceci évoque donc un contexte plein de glamour hollywoodien qui impressionne, qui en jette comme on dirait familièrement. L'informateur 3 nous livre lui-même la sémantique de sa proposition créative en la glosant pratiquement par le synonyme vulgaire şmecher qui lui est venu à l'esprit dès la première rencontre avec le mot sleek (dans notre corpus, à la ligne 16). On peut supposer que ce correspondant en langue cible lui est venu dès le départ certes avec l'ambiguïté du double sens que nous avons évoquée au départ de notre commentaire et qui était apparemment stockée dans son mental. Il semble l'avoir gardé en son for intérieur à la recherche d'une variante plus châtiée qu'il a finalement trouvée avec la métaphore ca scoase din cutie. La confirmation de cette hypothèse nous est fournie au moment où il demande nu poți să zici mașini șmechere [tu ne peux tout de même pas dire des voitures pour flamber, qui en jettent] (44-45) à quoi l'informateur 1 répond: $n u$, în nici un caz, că e prea colocvial [non, en aucun cas, parce que c'est trop familier] (45-46); ca scoase din cutie n'est donc que le correspondant châtié d'un verbum interius qu'il héberge en son for intérieur depuis la compréhension holistique du texte et qu'il s'est graduellement construite au cours de sa lecture, illustrant la thèse heideggerienne: Den Bedeutungen wachsen Worte zu, ainsi que la thèse cognitiviste que le sens se construit.

Mais à côté de ces éléments top down, qui tiennent donc du savoir culturel, les éléments bottom up jouent également un rôle dans le cheminement vers cette solution créative, comme nous le montre le dialogue qui suit. Comme il leur paraît évident que le qualificatif new qui suit immédiatement le mot sleek en question devra être traduit par noi, l'informateur 1 fait remarquer que dar nu poți să spui apoi și noi și scoase din cutie [mais tu ne peux dire après à la fois nouveau et sorti de la boîte], car l'énoncé serait tautologique en langue cible étant donné que ce qui est coase din cutie doit forcément être neuf. Et lorsque l'informateur 2 conteste cette logique (49), l'informateur 1 insiste en ajoutant que dacă l-ai scos din cutie, teoretic, trebuie să fie nou, cadoul [si tu l'as sorti de la boîte, théoriquement il doit être neuf, le cadeau]. Le contexte immédiat, bottom up, a donc également joué un rôle dans l'apparition de cette métaphore créative, par assimilation sémantique; le noi contigu a mis en évidence un des traits pertinents de la métaphore lexicalisée: dans le champ sémantique de la métaphore et de la scene qu'elle représente, l'un des éléments sémiques virtuels - la nouveauté - a été activé au contact du mot voisin et est devenu prototypique dans l'analyse qu'en fait l'informateur 1, qui veut l'exclure pour raisons de tautologie.

Ce qu'il est important de faire comprendre aux étudiants, c'est que la perception de l'informateur 1 est légitime; c'est un des processus qui font naître le sens au contact 
du lecteur. Il faut leur montrer que ce processus d'assimilation sémantique n'est que le pendant des phénomènes d'assimilation en phonétique, ou l'assimilation est plus palpable et prouvée par les sonagrammes.

La solution créative est donc le résultat d'une fusion des horizons top down du traducteur et bottom up du texte (pour marier la terminologie gadamérienne avec celle des cognitivistes), fusion qui a fait jaillir le sens. Mais, comme nous verrons plus bas, le processus de contamination par la contiguïté du mot new n'est qu'un exemple d'assimilation sémantique, soutenu, comme nous le verrons, par toute une isotopie de la nouveauté plus générale sous-jacente à l'ensemble du texte.

Si ca scoase din cutie convient aux trois autres informateurs, c'est que cette formulation métaphorique, aux contours sémantiques indécis, leur permet justement de récupérer beaucoup de choses (43) de façon nuancée (43) : ils semblent préférer une image nuancée, aux contours pas trop précis, pour rendre le maximum de choses, laissant au lecteur le soin de «meubler» cette métaphore sur son fond de vécu à lui. Sleek n'ayant pas pour eux un sens assez déterminé, ils préfèrent le rendre par une image aux contours flous.

\section{La traçabilité de cette trouvaille. Quels sont les éléments qui ont permis d'arriver à cette solution créative?}

Notre corpus montre que les facteurs qui ont contribué à cette solution créative sont fonction à la fois du comportement des informateurs (processus top down) et des caractéristiques de notre texte (bottom up). La fusion de ces deux éléments déclenche des processus cognitifs qui permettent de retracer le cheminement vers cette solution créative.

\subsection{L'horizon du traducteur marqué par la "Leibhaftigkeit» et les "somatics"}

Voyons d'abord ce que le corpus nous apprend sur leurs comportements traduisants. D’une façon générale, nos différents corpus de données révèlent qu'aucune tête de traducteur n'est vierge de théorie (Bălăcescu et Stefanink 2003a), malgré tout ce que les opposants aux théories peuvent prétendre. La maxime de traduction qui domine dans cet extrait de corpus, du début à la fin, est qu'il faut trouver en langue cible un mot qui réunisse tout ce qu'il y a dans le mot du texte source, soit linguistiquement parlant une idée jadis chère à Nida (1974: 52) qui prétendait que «What we do aim at is to translate the bundles of componential features». Ainsi nous trouvons au début de notre corpus: trebui un cuvânt care să le recupereze pe toate [il faut un mot qui récupère tout] (24-25) et à la fin nu văd un singur cuvânt care să redea tot [je ne vois pas un seul mot qui rende tout] (53-54). Au vu des réflexions qui égrènent leur conversation, cette maxime reste sous-jacente tout au long de leur négociation. La plupart des différentes propositions de traduction sont évaluées implicitement ou explicitement par rapport à cette maxime. Ainsi la remarque que l'anglais sleek est mai mult [bien plus] que le roumain elegant, ou que le roumain elegant est prea puțin [trop peu] (38-39) permet de conclure à une analyse implicite en traits pertinents, au service d'une «spot-the-difference-strategy», terme introduit par Krings (1986). Et lorsque, au cours de l'évaluation de la solution créative métaphorique trouvée, l'informateur 
1 estime que cette solution recuperează multe chestii [récupère beaucoup de choses] (42-43), il pense là encore, linguistiquement parlant, à la récupération des traits pertinents du mot source.

\subsubsection{Un élément déterminant: l'empathie avec le texte}

Ce qui est frappant, c'est que cette discussion sur ce qu'ils pensent être les traits pertinents du mot sleek, masque l'impact de ce que l'informateur 4 appelle «l'atmosphère du texte», qui détermine leurs choix traduisants. L'informateur 3 , qui trouvera la solution créative, est aussi celui qui a le plus d'empathie avec le texte, c'est lui qui trouve les étapes intermédiaires décisives qui y mèneront. C'est lui qui le premier exprime son insatisfaction ${ }^{11}$ par rapport à elegante (18), c'est lui qui a une démarche associative, créant ce que nous avons appelé un nuage sémantique, dans lequel il revient sur le mot anglais sleek pour l'associer au roumain moderne, essayant ainsi de créer un flow qui va l'entraîner dans un enchaînement associatif qui aboutira à la solution métaphorique. C'est lui qui, en réponse à lucios et strălucitoare, proposés par l'informateur 2 pour décrire le brillant des voitures, pose le regard sur le portefeuille du propriétaire (scumpe [cher] 23), opérant ainsi un déplacement de focus sur le modèle du figure / ground alignment de Langacker (1987), tel que le préconisent les défenseurs de la pensée latérale (de Bono 1970), stratégie qui, en l'occurrence, n’a pas d'aboutissement immédiat, mais montre une implication active de toute sa personne, avec son expérience top down, dans cette scene, implication qui va l'amener à continuer de centrer son attention sur le propriétaire de la voiture plutôt que sur celle-ci. S'il arrive à văcsuite c'est toujours avec le regard sur la personne qui astique sa voiture et la fait ainsi briller, plutôt que sur la voiture elle-même, et c'est dans cette même foulée qu'il aboutit à ultraspălate et ultraaranjate, qui proviennent de la même scene «soins de la voiture» et viennent renforcer de façon encore plus expressive le ultravăcsuite. Son comportement remplit parfaitement les critères mis en évidence par les chercheurs en créativité (Brodbeck 1995).

C'est cet investissement "corps et âme» dans le texte - la Leibhaftigkeit chez Paepcke (1986) ou la somaticité chez Robinson (1991) - qui lui permet de se mettre dans la peau de la situation et de la vivre. La priorité de sa perception émotionnelle du texte, par les sens, devient manifeste lorsqu'il avance intuitivement la proposition văcsuite [laquées], qui est loin d'être une aberration. Elle n'est que l'aboutissement des chaînes associatives stockées dans son bagage cognitif (world knowledge), qui impliquent la scene des soins que les nouveaux propriétaires de voitures prodiguent aux symboles de leur nouveau standing de vie. Mais son intellect censure sa trouvaille intuitive: sa pensée logique (convergente selon Guilford: 1975) n'a pas été en mesure de suivre sa perception intuitive par les sens. Et cette perception intuitive par les sens est légitime puisque, avec ses isotopies de brillance et de lumière, le texte fait largement appel aux sens.

Un dialogue avec le texte que les autres ne suivent qu'à une certaine distance, n'offrant comme contrepartie à son vocabulaire dynamique que des termes statiques, comme lucios, strălucitoare, etc. Sans parler de l'informateur 4 qui n'intervient pas du tout dans le dialogue et voit le texte «aus der Distanz des Zuschauers» [de la distance, en spectateur] comme dit Paepcke (1978: 99) ou en lançant des «bezele din avion » [baisers de l'avion] comme dit Mavrodin (1982/1998), évoquant le critique de traduction professionnel de la maison d'édition qui n'a pas le toujours temps de s'investir dans le texte pour acquérir l'empathie nécessaire à la saisie du sens. 


\subsubsection{La créativité peut-elle s'enseigner?}

Ce sont là les processus sur lesquels le didacticien de la traduction doit attirer l'attention de ses informateurs étudiants. Comme nous l'avons montré (Bălăcescu et Stefanink: 2003b), ce sont des processus mis en évidence par les chercheurs cognitivistes comme ayant une certaine cohérence. Ce sont ces processus qui sont à la base de la traçabilité des solutions créatives, auxquelles l'approche herméneutique mène de façon quasi contraignante de par ses fondements. La première tâche du didacticien de la traduction c'est de faire prendre conscience aux apprenants que ce potentiel créatif est en eux, sa seconde tâche étant de trouver les moyens pour l'améliorer après cette prise de conscience. Vu le caractère cohérent de ce dispositif - qui mène de la scenes and frames semantics de Fillmore, aux chainings associatifs de Lakoff (1987), en passant par la sémantique des prototypes de Rosch (1973), le figure-ground alignment de Langacker, le lateral thinking de de Bono et les TOPs et MOPs, dans la «dynamic memory» de Schank (Bălăcescu et Stefanink: 2003b) -, un apprentissage sous forme de sensibilisation est une entreprise tout à fait légitime et prometteuse, au grand dam de ceux qui ne voudraient voir dans l'approche herméneutique que de «vagues hypothèses» (Kußmaul 2000: 59) ou des processus «nébuleux» (Nord 1995: 42). Il ne s'agit évidemment pas d'enseigner des structures algorithmiques qui permettraient d'aboutir à des solutions créatives, mais d'agir sur les comportements favorisant cette créativité et d'apprendre à créer un environnement qui lui soit favorable. Les chercheurs en créativité se basent pour cela sur les recherches en sciences cognitives, à commencer par le modeste mais combien révélateur Metaphors we live by (Lakoff et Johnson 1980).

Voyons maintenant ce qui se cache derrière l'expression «atmosphère du texte».

\subsection{L'horizon du texte: les isotopies et leur impact sur les choix traduisants dans notre corpus de données. Nihil ex nihilo!}

Que dire d'un terme comme "atmosphère» (60), aux contours sémantiques vagues? C'est à cet endroit que la linguistique du texte nous fournit un instrument fiable (dans le sens d'une objectivation de ce terme impressionniste) avec la notion d'isotopie. En effet, cette «atmosphère» que ressentent nos informateurs est le résultat d'un réseau sémantique créé par les isotopies du texte et, si nos informateurs n'arrêtent pas de répéter, comme un mantra, leur besoin de «trouver un mot qui rende tout», c'est sous la pression sémantique exercée par les isotopies du texte, et le «tout» qu'ils s'efforcent de rendre n'est pas l'ensemble des traits pertinents du mot sleek, mais l'ensemble de toutes les impressions recueillies au cours de leur lecture d'un texte qui fait largement appel aux sens.

Il y a, dans un texte, des mots qui ont ce que Wittgenstein (1953) a appelé des Familienähnlichkeiten [ressemblances familiales] et qui s'éclairent et se conditionnent mutuellement du point de vue sémantique. Dans notre texte par exemple, les mots lucios, strălucitoare, uns, văcsuite ont en commun le fait d'évoquer quelque chose de brillant. Greimas (1966) a appelé l'ensemble des mots, qui étaient ainsi reliés entre eux, des isotopies. Pour Greimas, à l'époque du structuralisme linguistique, le lien entre les chaînons d'une isotopie était établi sur la base de sèmes communs. Peu à peu la notion d'isotopie s'est étendue à d'autres éléments porteurs de sens ${ }^{12}$, de sorte que nous dirions volontiers que les isotopies sont des "guides de compréhension». 
Les éléments d'une telle isotopie sont connectés entre eux et forment un réseau. Ce sont ces multiples interconnexions qui font naître un sens qui n'est pas lié à un mot particulier, mais dont participent tous les mots de l'isotopie en question. Ce conditionnement est possible sur la base de ce que les chercheurs en sciences cognitives nous ont appris. Le sens qui se reflète dans les propositions de traduction de nos informateurs est non pas la traduction de «bundels of componential features» du mot sleek, mais l'aboutissement d'une cristallisation lexicale d'un sens diffus dans le texte sous la pression sémantique exercée par les isotopies. Quelles sont donc ces isotopies?

\subsubsection{Isotopie de l'opulence}

Le texte entier est dominé par une isotopie générale de l'opulence, qui est représentative de l'immense étonnement des Allemands de l'Est confrontés aux «splendeurs» de l'Allemagne de l'Ouest. Image d'opulence qui est soutenue par des observations de détails, comme par le fait que l'Opéra de Dresde est non seulement reconstruit, mais plein à craquer: Every seat is filled at Dresden's magnificent neo-classical opera house. Et cette plénitude n'est pas seulement évoquée par un adjectif abstrait comme le serait full ou une phrase abstraite qui nous informe que l'Opéra a de nombreux visiteurs, mais nous voyons littéralement les «volumineux» nouveaux riches, plein de gigantic pastries, remplir de leur opulente masse corporelle jusqu'au dernier des sièges. Ainsi l'ensemble du texte est rempli d'une multitude de détails qui viennent soutenir cette atmosphère d'opulence, notamment par sa richesse en adjectifs qui quelle que soit la sous-isotopie dont ils relèvent contiennent toujours des sèmes collatéraux soutenant l'idée d'opulence générale.

Quelles sont ces sous-isotopies qui viennent soutenir l'idée d'opulence générale?

\subsubsection{La sous-isotopie de paillettes et de brillance}

Des propositions de traduction comme strălucitoare [étincelante] (17), lucios [brillant] (18, 30), lucioase [brillante] (30), mă duce cu gândul la ceva uns [me fait penser à quelque chose de oint] (30), văcsuite, văcsuit [ciré, reluisant] $(28,34,31)$ peuvent être dues à des adjectifs comme shiny (service stations), gleaming (self-service restaurants), magnificent, qui forment la sous-isotopie de paillettes et de brillant pouvant aussi avoir contribué à l'évocation de la scène où l'on fête les vedettes avec tout le clinquant qui les entoure dans les grands spectacles télévisés, où on les décrit $\underline{c a}$ scoase din cutie [comme sortis de la boîte à cadeaux].

\subsubsection{La sous-isotopie de confort et richesse}

Très proche de cette sous-isotopie de brillance on trouve la sous-isotopie de confort, explicitement représentée par le mot comfortable (burghers), ainsi que par un certain nombre d'autres mots liés à cette scène du confort. C'est ainsi que l'auteur de l'article souligne l'idée de confort en choisissant, dans le paradigme des mots à sa disposition pour désigner les habitants de Dresden, le mot - sémantiquement marqué - de burgher et non pas citizen. La même connotation de confort est véhiculée par les well-dressed women [...] who gather for creamy coffee and gigantic pastries, tout le scénario étant couronné par le French champagne, que ces messieurs-dames ne sont pas en train de vulgairement boire, mais auquel ils touchent avec distinction du 
bout des lèvres, sipping un champagne, qui ne peut être que français et qui est communément connoté avec l'idée de succès. Ce sont assurément là les scènes qui font penser à l'idée de confort (26) et qui déclenchent des propositions comme conforta-

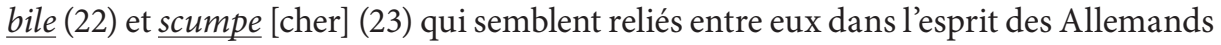
de l'Est tels que les voient nos informateurs, cette dernière proposition étant sans doute déclenchée par la visualisation des well-stocked convenience stores.

\subsubsection{La sous-isotopie du neuf et de l'impeccable}

Ce qui donne cette idée de nouveauté - qui entraîne même nos informateurs à vouloir traduire sleek par noi-nouțe, un redoublement de noi, qui peut se traduire par flambant neuf et qui a certainement contribué à l'idée de l'image ca scoase din cutie - c'est que dès la première ligne nous sommes sous l'impression de mots qui évoquent la nouveauté, tantôt explicitement, comme le new qui suit immédiatement sleek, tantôt plus subtilement, lorsque l'auteur choisit repaved et straightened au lieu de paved et straight, indiquant un processus de rénovation. Mais l'idée du neuf et d'impeccable n'est pas limitée à ces mots, elle se retrouve à d'autres endroits du texte comme dans l'image des well-dressed women ou du magnificent neo-classical opera house; et ce d'autant plus lorsqu'on sait que Dresden avait été détruite à $80 \%$ lors de la Deuxième Guerre mondiale et que, lors de la reconstruction de la ville, l'opéra a été l'objet d'une attention toute particulière ${ }^{13}$. Cette isotopie donne lieu à un certain nombre de propositions comme noi-nouțe [flambant neuf] (49), ireproşabile (50), ultramoderne $(54,55)$, ultraspălate [ultralavés] (31), ultraaranjate (31).

\subsubsection{La sous-isotopie du gigantisme et de l'anthropomorphisation}

Nous avons vu jusque là des propositions de traduction qui se laissent rattacher à des mots évoquant le confort, le neuf, la propreté, le brillant, etc. que l'on trouve sémantiquement assez apparentés aux mots du texte source. Ceci vaut aussi pour les mots du texte source qui évoquent le gigantisme, comme enormous supermarkets, shopping emporiums, giant cranes. Cette isotopie a certainement contribué à créer une sémantique de l'exagération qui traverse le texte.

Par contre, comment expliquer des propositions comme extravagante (25), sinon par l'atmosphère fantasmagorique qui est créée par une combinaison de l'isotopie du gigantisme et de l'isotopie de l'anthropomorphisme, que l'informateur 1 ressent bien quand il trouve que la proposition ultramoderne [...] are o nuanță de extravaganță [...] care nu e prezentǎ în text neaparat [contient une nuance d'extravagance qui n'est pas forcément dans le texte] (55-56). L'argumentation avec laquelle l'informateur 1 écarte ultramoderne au profit de ca scoase din cutie est révélatrice: en argumentant que ultramoderne n'est pas présent dans le texte, il implique que la métaphore $\underline{c a}$ scoase din cutie, quant à elle, serait présente dans le texte. Apparemment il la sent présente, même si ce n'est pas sous une forme verbalement matérialisée. Il dit donc implicitement que cette métaphore, au contraire d'ultramoderne, " est présente dans le texte», sachant pertinemment bien que ce n'est pas sous une forme matérialisée verbalement. On ne peut reconnaître plus explicitement - et ceci dans une naïveté inconsciente qui donne tout son poids à ce témoignage - qu'on traduit ce qui est «entre les lignes» pour rappeler les mots de Schleiermacher (1977: 315 ).

Gigantisme et anthropomorphisme sont entrelacés dans des images comme enormous supermarkets, shopping emporiums, giant cranes. Les grues ne sont pas 
simplement big, donc grandes, elles sont giant, donc gigantesques, et elles ne sont pas simplement là, debout, pour quoi stand aurait suffi, non elles stand tall against [!] the sky. Il y a un certain dynamisme, une certaine agressivité dans cette image, nourrie surtout par le mot against; on imagine ces grues comme des gratte-ciels, véritablement en train d'étendre leurs bras et mains dans la pénombre d'un ciel d'orage menaçant, entrecoupé d'éclairs lancés par les shining service stations et les gleaming self-service restaurants. Et les enormous supermarkets, les shopping emporiums ne sont pas simplement là, immobiles, non ils dot [!] the landscape, comme des champignons qui sortent après la pluie. Et les shiny service stations ne sont pas non plus simplement là, non, elles come [!] equipped with well-stocked convenience stores and gleaming self-service restaurants; même s'il s'agit d'une formulation idiomatique sans intention de décrire un mouvement, la valeur prototypique associée avec le verbe come est bien celle d'un mouvement, donc d'anthropomorphisation des shiny service stations, même si l'impact est moins fort que celui des grues géantes qui stand tall against the sky. Et ce dynamisme anthropomorphisant est communiqué dès la première phrase: d'emblée nous sommes confrontés aux voitures (et pas aux chauffeurs) qui speed, c'est-à-dire foncent à toute allure sur les autoroutes rénovées. Et ce dynamisme anthropomorphisant est contagieux: dans cet environnement sémantique d'un activisme fébrile, le gleaming des self-service restaurants perd son caractère d'adjectif décrivant un état, au profit de sa nature originelle de participe présent décrivant l'activité des self-service restaurants qui tout à coup rayonnent activement, répandant la lumière autour d'eux. Celui qui a l'empathie nécessaire pour vivre ce texte ressent une sorte d'atmosphère surnaturelle qui se cristallise dans la proposition extravagante bien que, comme constate l'informateur 1 , le mot ne soit pas dans le texte.

\subsection{La cristallisation du sens diffus: évaluation et plausibilité de notre approche}

Dans son essai intitulé De l'amour, Stendhal (1822) décrit le phénomène de cristallisation comme un processus qui peut amener un amoureux enthousiaste à prêter par exagération, à la femme aimée, des traits qu'elle ne possède pas dans la réalité. C'est ce qui semble se produire chez nos informateurs, du moins dans la mesure où ils pensent traduire strictement le mot sleek (même si c'est sous la pression sémantique des isotopies qui traversent le texte). Ils abordent le texte comme le préconise Gadamer, en «entrant dans le jeu», par lequel ils se laissent entraîner de plus en plus jusqu'à perdre le contrôle de leurs mots, utilisant les mots, mais se laissant aussi dicter par les mots.

Notre analyse ne signifie pas que, dans le cas précis que nous venons d'étudier, nous privilégions forcément la solution métaphorique choisie. Nous voulons simplement montrer qu'elle a sa justification dans le sémantisme qui traverse tout le texte. Nous assistons certainement à un processus de cristallisation au cours duquel les participants au dialogue, sous l'influence d'un texte qui fait fortement appel aux sens, investissent ce premier mot du texte de toutes ces impressions recueillies au fil de leur lecture. Du point de vue didactique l'essentiel est de leur en faire prendre conscience; rien de mieux pour cela que le livre de Schwarz-Friesel (2007) intitulé Sprache und Emotion [Langage et émotion], qui met en évidence la priorité de la saisie émotionnelle d'un texte sur l'analyse linguistique. 
Cette traduction trouverait aussi sa légitimation du point de vue des recherches sur la créativité en traduction, telles que les a développées Paul Kußmaul (2000), notamment au chapitre 7 (pp. 150-188), où il établit une typologie des processus cognitifs récurrents qui mènent à des solutions créatives récurrentes qui sont explicables et légitimées par les recherches en sciences cognitives. Dans notre cas, la solution trouvée par nos informateurs tombe dans la catégorie "Einrahmung» [encadrage] (pp. 185-188). Nous avons, en effet, affaire à plusieurs éléments du scénario «opulence» (tous les éléments qu'on trouve dans les sous-isosopies: brillance, gigantisme, perfection, etc.) qui sont eingerahmt [encadrés] dans un frame (au sens fillmorien du terme), c'est-à-dire un mot ou une expression qui peut être, comme dans notre cas, une tournure métaphorique lexicalisée.

Nous avons de toute façon ici un bel exemple de Horizontverschmelzung même si la fusion ne se fait pas tout à fait, comme l'entendait Gadamer, entre le lecteur et l'auteur du texte, mais plutôt, dénuée de l'historicité caractéristique de la conception gadamérienne, entre le lecteur et le texte, comme le voulait Paul Ricoeur, pour qui le sens ne se trouve ni derrière le texte ni dans la pensée de son auteur, mais dans ce que Ricoeur (1975: 156) nomme «l'orient du texte».

\section{Conclusion : que nous révèle cette étude?}

D’une façon générale, cette étude empirique montre que l'approche herméneutique épouse bien la réalité du traducteur. Ce que nous avons appelé "cristallisation» illustre très bien la façon dont Stolze (2011a: 129) décrit le processus de traduction quand elle parle de "gradual, autopoietic process of 'finding words' for ideas which are already there». Cette cristallisation illustre également de façon pertinente la thèse de Heidegger (1927/1967: 160): «Den Bedeutungen wachsen Worte zu» [Les mots viennent (dans un lent mouvement de croissance) aux significations].

Et finalement cette cristallisation du sens illustre aussi l'affirmation de Gadamer, citée à l'entrée de notre étude et concernant le verbum interius: le sens que le traducteur a dégagé dans son mental à partir du dialogue avec le texte est son verbum interius, pour lequel il essaye de trouver les mots. La fameuse Stimmigkeit (Stolze: 2003) de la traduction n'est pas la correspondance entre les traits sémantiques clés du texte source et du texte cible ${ }^{14}$, mais la correspondance entre ce verbum interius et le texte en langue cible que le traducteur est en train de créer, comme nous l'avons expliqué ailleurs (Bălăcescu et Stefanink: 2012).

La démarche utilisée dans cette étude nous a permis de mettre en évidence le bien-fondé d'un certain nombre de thèses avancées par les tenants de l'approche herméneutique en traduction.

- Elle nous a permis de satisfaire aux besoins de Thomas l'incrédule à la recherche des preuves «tangibles» de l'existence d'un sens qui «flotte» entre les lignes ou derrière les mots: ce sens existe bel et bien entre les isotopies du texte et traverse le texte comme un brouillard qui se lève à partir des mots aux contours flous, que la sémantique des prototypes nous a révélés, et dont les contours s'estompent encore davantage dans ce brouillard sémantique ${ }^{15}$, dû à leur conditionnement réciproque. Et de même que ce brouillard se condense et retombe sous forme de gouttelettes, de même ce sens se cristallise et retombe sous forme de mots qui ne sont pas forcément les correspondants plus ou moins proches des mots du texte source. Le sens n'est pas là de façon statique dans l'attente d'être découvert, «mais il est dans ce que Ricoeur 
nomme 'l'orient du texte', c'est-à-dire le monde du texte qui se lève dans l'interprétation» (Grondin 2013: 94).

- Cette approche du sens est légitimée par les recherches cognitivistes sur les processus bottom up et top down comme constitutifs du sens.

- Contrairement au lecteur normal, par qui ce sens est perçu plus ou moins intuitivement et inconsciemment, le traducteur doit expliciter ce sens - le Auslegen [explicitation] heideggerien - pour le traduire.

- Les différentes propositions de traduction sont des manifestations de cette Auslegung par nos informateurs / traducteurs. Elles sont des explicitations tentatives - les TUÄ (Tentative Übersetzungsäquivalente de Krings 1986) - de leur verbum interius, qui est le sens du texte tel qu'il s'est construit et continue de se construire dans leur mental.

- Notre corpus de données conversationnelles montre clairement que, malgré leurs timides tentatives d'analyse stylistique ou logique, nos informateurs ont une approche holistique du texte.

- Dans cette approche ils sont subjugués par les impressions physiques, notamment visuelles, ce qui vient confirmer les théories de Paepcke sur la Leibhaftigkeit du traducteur et celles de Robinson sur la somatique du traducteur.

- Notre appel à la linguistique du texte d'une part et aux résultats des recherches en sciences cognitives d'autre part, nous a permis de rendre traçables et de légitimer ces cristallisations d'un sens diffus entre les mots du texte.

- Cette traçabilité obéit aux critères de scientificité définis par Popper (1935: 7- 8) quand il écrit, à propos de la «trouvaille» de l'inventeur, dans un chapitre intitulé «Die deduktive Überprüfung der Theorien» que cette trouvaille n'est certes pas prévisible comme résultat d'une méthodologie appliquée algorithmiquement (comme le voudrait Gerzymisch 2013), mais satisfait aux exigences de scientificité dans la mesure où le chemin qui a mené à elle est traçable et remplit ainsi le critère de «transparence» mis en avant par les objectivistes.

- La thèse d'un sens qui se dégage d'entre les lignes du texte au contact du traducteur condamne le traducteur à la créativité conçue comme une problem solving activity (Guilford 1975).

- Cette créativité est fondée sur un certain nombre de processus de nature associative mis en évidence par les recherches en sciences cognitives.

\section{9. Épilogue}

Paul Kußmaul, auquel nous avions demandé la permission d'utiliser son texte, nous a demandé de lui envoyer l'article que nous étions en train d'écrire, ce que nous avons fait avec une certaine appréhension, ayant à l'esprit les critiques qu'il avait formulées jadis à l'égard de l'approche herméneutique, la taxant de «Mystifizierung" [mysticisme] et lui reprochant son caractère «vorwissenschaftlich» [pré-scientifique], ainsi que sa terminologie "unbestimmt» [vague], qui se perdait dans des "raunende Andeutungen» [hypothèses peu claires] (Kußmaul 2000: 59).

Quelle ne fut pas notre surprise à la lecture de sa réponse:

[...] votre interprétation très différenciée du protocole des informateurs m’a beaucoup impressionné. Si c'est ainsi que l'on comprend l'approche herméneutique, combinée avec les modèles de la linguistique du texte et de la sémantique cognitive, comme vous l'avez fait, elle est absolument convaincante. Avec votre aide j'ai vu beaucoup de subtilités du texte que je n'avais pas encore remarquées jusque là. Bien cordialement, Paul Kußmaul (courriel du 20 octobre 2015). 
La boucle était bouclée. Kußmaul - traductologue et chercheur en créativité, qui connaissait intimement ce texte pour lui avoir consacré une partie de son livre Kreatives Übersetzen - correspondait bien au profil de l'expert (Risku 1998) susceptible d'établir cette "vérité consensuelle», chère a Habermas (1983), qui doit tenir lieu d'une objectivité impossible à atteindre dans les sciences humaines. À la place de cette objectivité, Husserl pose que "durch wechselseitige Kritik geläuterte und jeder Kritik standhaltende Resultate» [des résultats éprouvés et affinés par une critique mutuelle et résistants à toute critique] ont valeur de vérité objective (Husserl 1969/1977: 7).

\section{REMERCIEMENTS}

Cet article doit beaucoup à l'interaction de Bernd Stefanink avec les étudiants de son séminaire «Traduction et Herméneutique», dans le cadre de sa mission de professeur invité à l'Universidade Federal de Santa Catarina (UFSC). Cette mission a été commanditée par la Fondation Johann Gottlieb Herder (en collaboration avec le DAAD) qui permet aux professeurs émérites des universités allemandes de disséminer leur savoir à l'étranger. Nous remercions la Fondation Herder pour le soutien financier, ainsi que la Professeure Dr Andreia Guerini, qui a formulé cette invitation, et les participants au séminaire. Je remercie aussi le DAAD qui a commandité ma mission à l'Université de Craiova (création d'un institut de traductologie) au cours de laquelle j’ai pu recueillir le corpus traductif qui a servi de base à cette étude.

\section{NOTES}

1. Traduction qui ne rend malheureusement pas l'aspect dynamique du wachsen zu, qui implique une «lente croissance vers», donc littéralement: «les mots croissent vers le sens», qui, lui, est là avant les mots. Cette phrase de Heidegger nous semble fidèlement décrire le travail du traducteur qui se construit sa (!) représentation mentale du sens du texte - qui devient le verbum interius de Saint-Augustin - à laquelle doivent venir les mots.

2. On trouvera une discussion détaillée dans Stefanink et Bălăcescu 2014.

3. Aussi, c'est en 1993 dans sa préface à L’universalité de l'herméneutique que Gadamer écrira que « [c]'est le mérite insigne de Grondin d'avoir fait ressortir en quoi ce dialogue 'intérieur' constitue le véritable fondement de l'herméneutique, pour lequel j'avais pu, dans Vérité et méthode me réclamer d'Augustin [...]» (Grondin 1993: V).

4. Par opposition à actus signatus, distinction scolastique, reprise par Heidegger et à sa suite par Gadamer et Grondin. L'identification du actus exercitus avec le verbum interius entreprise par Gadamer peut sembler abusive (Vessey 2011). Heidegger se garde de l'attribuer à Saint-Augustin, se limitant à le situer dans la scolastique.

5. Texte peut-être moins connu à l'étranger, mais bien connu de tous les lycéens allemands en terminale, du moins du temps de Stefanink où ce texte figurait au programme de lecture.

6. O'Keeffe (2015: 168) décrit le processus de traduction «as a process [...] always on the way to a possible future completion».

7. Le terme est de Lenk (2014: 81) il s'agit d'une «Spezialform des interaktiven Interpretierens» qu'il appelle imprégnation [Imprägnieren]: "L'imprégnation telle que nous la concevons est en fait quelque chose qui relie l'apport du monde réel à un processus structurant dans lequel nous 'entrons' nous-mêmes; et les deux sont imbriqués l'un dans l'autre» (notre traduction), le monde réel étant dans notre cas le texte à traduire.

8. La méthode a été exposée dans Stefanink 1995; Cercel (2013: 141-145) en fournit une évaluation (positive).

9. Â cet endroit, deux mots sur la notion de «dialogue» chez Gadamer: contrairement à ses prédécesseurs, notamment Wilhelm Dilthey, qui ont attiré l'attention sur les «préjugés» dont nous sommes victimes dans la perception de la réalité, Gadamer a attribué une valeur positive à ces préjugés, à condition d'en prendre conscience et de les intégrer dans la recherche de la "vérité» (dans notre cas du sens du texte). Cette prise de conscience doit se faire dans le dialogue avec autrui, dans un esprit d' «ouverture», dans lequel nous offrons l'«hospitalité» à la pensée de l'autre au 
sens où l'entendent le philosophe Paul Ricoeur ou le traductologue Antoine Bermann. Retenons que ces préjugés sont par nature incontournables (Lenk 2014) parce qu'ils sont le résultat de notre vécu et du réseau engrammatique qui en résulte dans notre mental (Stefanink et Bălăcescu : 2015).

10. Cela rejoint et vient soutenir l'isotopie de l'anthropomorphisme qui traverse le début du texte.

11. L'insatisfaction est considérée par les chercheurs en créativité comme le premier pas vers la créativité.

12. Ainsi on peut constater qu'un texte est traversé, par exemple, par une isotopie de l'ironie (Stefanink et Bălăcescu: 2015).

13. Notons, en passant que c'est là le type d'information que le traducteur herméneute doit recueillir pour aboutir à ce que nous appellerions une «compréhension motivée» ou «fondée sur l'expertise » (grounded understanding chez Stolze 2011a: 75).

14. Ce que soutenait Stolze jadis, et comme on peut encore le trouver dans Stolze 2011b, sans doute par mégarde, comme un fossile datant de Stolze 1992. Stolze (2015) s'est ralliée à notre compréhension du terme.

15. En utilisant ce langage métaphorique, nous nous réclamons des chercheurs cognitivistes qui ont reconnu une valeur épistémologique aux métaphores.

\section{RÉFÉRENCES}

BǍLĂCESCU, Ioana et STEFANINK, Bernd (2003a): Une approche théorique pour la traduction. In: Ghjacumu Thiers, dir. Baratti. Commentaires et réflexions sur la traduction de la poésie. Collection «Isule Literarie. Des îles littéraires». Albiana - Bu - Ccu - Iitm, 24-77.

BĂLĂCescu, Ioana et Stefanink, Bernd (2003b) : Modèles explicatifs de la créativité en traduction. Meta 48(4):509-525.

BĂLĂCESCU, Ioana et STEFAnink, Bernd (2012): De la valeur heuristique du terme dans l'approche herméneutique. In: Larisa CERCEL et John STANLEY, dir. Unterwegs zu einer hermeneutischen Übersetzungswissenschaft. Radegundis Stolze zu ihrem 60. Geburtstag. Tübingen: Narr, 224-238.

BRodBeck, Karl-Heinz (1995): Entscheidung zur Kreativität. Wege aus dem Labyrinth der Gewohnheiten. Darmstadt: Wissenschaftliche Buchgesellschaft.

CERCEL, Larisa (2013): Übersetzungshermeneutik. Historische und systematische Grundlegung. St. Ingbert: Röhrig.

Damasio, Antonio (1994) : Descartes' Error: Emotion, Reason and the Human Brain. New York: Avon.

DE Bono, Edward (1970): Lateral Thinking. A Textbook of Creativity. London: Ward Lock Educational.

DEX, Dicționarul Explicativ al Limbii Române, București: Academia Română, Institutul Lingvistic 'Iorgu Iordan - Al. Rosetti'

Durieux, Christine (2009): Vers une théorie décisionnelle de la traduction. Lisa VII (3):349-367.

Fillmore, Charles (1976): Scenes-and-Frames Semantics. In: Antonio Zampolli, dir. Linguistic Structures Processing. Amsterdam: N. Holland, 55-88.

Gadamer, Hans-Georg (1960): Wahrheit und Methode. Grundzüge einer philosophischen Hermeneutik. Tübingen: J.C.B. Mohr.

GARFINKeL, Harold (1984): Studies in Ethnomethodology. Cambridge: Blackwell.

Gerzymisch-Arbogast, Heidrun et Mudersbach, Klaus (1998): Methoden des wissenschaftlichen Übersetzens. Tübingen: Francke

Gerzymisch, Heidrun (2013): Translation als Sinngebung. Münster: LitVerlag.

Greimas, Algirdas Julien (1966): Sémantique structurale. Recherche de méthode. Paris: Larousse.

Grondin, Jean (1993): L'universalité de l'herméneutique. Préface de H.-G. Gadamer. Paris: PUF.

Grondin, Jean (1991/2012): Einführung in die philosophische Hermeneutik. Darmstadt: Wissenschaftliche Buchgesellschaft.

Grondin, Jean (2013) : Paul Ricoeur. Que sais-je? Paris: Presses Universitaires de France.

Guilford, Joy Peter (1975): Creativity: A Quarter Century of Progress. In: Irving A. TAYlor et Jacob W. Getzels, dir. Perspectives in Creativity. Chicago: Aldine, 37-59. 
Habermas, Jürgen (1983): Moralbewußtsein und kommunikatives Handeln. Frankfurt am Main: Suhrkamp.

Hermans, Theo (2015): Schleiermacher and Plato, Hermeneutics and Translation. In: Larisa Cercel et Adriana Şerban, dir. Friedrich Schleiermacher and the Question of Translation. Schleiermacher Archiv (25). Berlin: De Gruyter, 77-106.

Heidegger, Martin (1927/ $/ 11967)$ : Sein und Zeit, Tübingen: Niemeyer.

Husserl, Edmund (1969/1977): Cartesianische Meditationen. Hamburg: Felix Meiner Verlag. IONEsCu, Tudor (2004): Călătorind spre capătul traducerii. In: Tudor IoNEsCu : Urme de condei. Cluj : Limes, 5-26.

KRINGs, Hans P. (1986): Was in den Köpfen von Übersetzern vorgeht. Eine empirische Untersuchung zur Struktur des Übersetzungsprozesses an fortgeschrittenen Französischlernern. Tübingen: Narr.

KLEIST, Heinrich von (1805): Über die allmähliche Verfertigung der Gedanken beim Reden. http://gutenberg.spiegel.de/buch/-589/1, consulté le 07/07/2017.

Kussmaul, Paul (2000): Kreatives Übersetzen. Tübingen: Stauffenburg.

LAKoff, George et Johnson, Mark (1980) : Metaphors We Live by. Chicago: University of Chicago Press.

Lakoff, George (1987): Women, Fire and Dangerous Things. What Categories Reveal about the Mind. Chicago: University of Chicago Press.

LANGACKer, Ronald W. (1987) : Foundations of Cognitive Grammar. Stanford: Stanford University Press.

LENK, Hans (2014): Methodologischer Schemainterpretationismus: Erkenntnistheoretisches zur Einführung. In: Gregor PAul, dir. Transkulturelle Logik. Universalität in der Vielfalt. Bochum-Freiburg: Projektverlag, 71-98.

Mavrodin, Irina (1982/1998²): Poietică şi Poetică. Craiova: Scrisul Românesc.

NidA, Eugene (1974): Semantic Structure and Translating. In: Wolfram Wills et Georg Thome, (édts), Aspekte der theoretischen sprachbezogenen und angewandten Übersetzungswissenschaft II. Heidelberg: Groos.

Nord, Christiane (1995): Recension de Radegundis STOLzE: Übersetzungstheorien. Eine Einführung. $6^{e}$ édition. Tübingen: Narr. Lebende Sprachen, 2:40-42.

O'Keeffe, Brian (2015): Prologue to a Hermeneutic Approach to Translation. In: Radegundis Stolze, John Stanley et Larisa Cercel (édts), Translational Hermeneutics. The First Symposium. Bucarest: Zeta Books, 145-176.

Oliva, Mirela (2009), Das innere Verbum in Gadamers Hermeneutik, Tübingen: Mohr Siebeck.

Paepcke, Fritz (1978): Übersetzen als hermeneutischer Entwurf. In: Fritz PaepcKe (1986). Im Übersetzen leben. Übersetzen und Textvergleich. Tübingen: Narr, 86-101.

Paepcke, Fritz (1981): Übersetzen zwischen Regel und Spiel. In: Fritz Paepcke (1986). Im Übersetzen leben. Übersetzen und Textvergleich. Tübingen: Narr, 121-134.

Paepcke, Fritz (1986): Im Übersetzen leben. Übersetzen und Textvergleich, édité par Klaus Berger et Hans-Michael Speier. Tübingen: Narr

Platon (1822-1840): Cratyle, dans Platon, Euvres complètes,Traduction par Victor Cousin, tome 11. Paris:Rey et Gravier

Popper, Karl, R. (1935): Logik der Forschung. Wien: Springer.

Ricoeur, Paul (1975): La Métaphore vive. Paris: Seuil.

RIsku, Hanna (1998): Translatorische Kompetenz: Kognitive Grundlagen des Übersetzens als Expertentätigkeit. Tübingen: Stauffenburg.

Robinson, Douglas (1991): The Translator's Turn. Baltimore: Johns Hopkins University Press.

Rosch, Eleanor (1973): Natural categories. Cognitive Psychology. 4:328-350.

SChleiermacher, Friedrich (1977): Hermeneutik und Kritik. Mit einem Anhang sprachphilosophischer Texte Schleiermachers. Frankfurt: Suhrkamp.

Schank, Roger C. (1982): Dynamic Memory. A Theory of Reminding and Learning in Computers and People. London/New York: Cambridge University Press. 
Schwarz-Friesel, Monika (2007): Sprache und Emotion, Tübingen: Narr Francke Attempto Verlag.

Stefanink, Bernd (1995): L'ethnotraductologie au service d'un enseignement de la traduction centré sur l'apprenant. Le langage et l'homme. 4:265-293.

Stefanink, Bernd (1997): 'Esprit de finesse' - 'Esprit de géométrie': Das Verhältnis von 'Intuition' und 'übersetzerrelevanter Textanalyse’ beim Übersetzen. In: Rudi KelLeR, dir. Linguistik und Literaturübersetzen. Tübingen: Narr, 161-184.

Stefanink, Bernd (2000): Analyse conversationnelle et didactique de la traduction. Studia Romanica Posnaniensia. XXV/XXVI: 283-298.

Stefanink, Bernd (2011): Un herméneute dans l'âme: Tudor Ionescu. RIELMA. 4:17-27.

Stefanink, Bernd et BăLĂcescu, Ioana (2014): Recension de Gerzymisch, Heidrun (2013): Translation als Sinngebung. Münster: LitVerlag Lebende Sprachen 59(1):188-207.

STEFANINK, Bernd et BĂLĂCESCU, Ioana (2015): Les cheminements de la créativité en traduction. Meta 60(3):599-620.

Stendhal ( $\left.{ }^{1} 1822 / 1980\right)$ De l'amour, édité en 1980 par Victor del Litto. Paris: Gallimard.

Stоlze, Radegundis (1992): Hermeneutisches Übersetzen. Linguistische Kategorien des Verstehens und Formulierens beim Übersetzen. Tübingen: Narr.

Stolze, Radegundis (2003): Hermeneutik und Translation. Tübingen: Narr.

Sтоlze, Radegundis (2011a): The Translator's Approach - Introduction to Translational Hermeneutics. Theory and Examples from Practice. Berlin: Frank und Timme.

Stolze, Radegundis ( $\left.{ }^{6} 2011 b\right)$ : Übersetzungstheorien. Eine Einführung. $6^{\text {e }}$ édition. Tübingen: Narr.

STOLZE, Radegundis (2015): Hermeneutische Übersetzungskompetenz. Grundlagen und Didaktik. Berlin: Frank und Timme.

Vessey, David (2011): Gadamer, Augustine, Aquinas, and Hermeneutic Universality. Philosophy Today 55(2):158-165.

Wittgenstein, Ludwig (1953): Philosophische Untersuchungen - Philosophical Investigations (D-E) Volume I. Oxford: Basil Blackwell.

\section{ANNEXE}

\begin{tabular}{|c|c|c|}
\hline 14 & 3: început sunteți de acord? & 3: commençons, vous êtes d'accord? \\
\hline & $2: \mathrm{da}$ & 2: oui \\
\hline & 1: sleek un cuvânt & $1:$ sleek un mot \\
\hline \multirow[t]{3}{*}{15} & 1: şmecher, da & 3: traître \\
\hline & 3: şmecher & 1: traître, oui \\
\hline & 4: maşini noi şi elegante & 4: des voitures neuves et élégantes \\
\hline 16 & $\begin{array}{l}\text { 3: cred că sleek vouă cum vi se pare? nu e } \\
\text { mai mult decat elegant }\end{array}$ & $\begin{array}{l}\text { 3: je pense que sleek... qu'est-ce que vous en } \\
\text { pensez? N'est-ce pas beaucoup plus } \\
\text { qu'élégant? }\end{array}$ \\
\hline \multirow[t]{2}{*}{17} & 1: nu ştiu da, e un & $1:$ je ne sais pas, mais c'est un \\
\hline & 2: strălucitoare? & 2 : brillant? \\
\hline \multirow[t]{2}{*}{18} & 1: termen formal & 1: terme formel \\
\hline & 2: păi sleek înseamnă lucios & $2:$ ben, sleek veut dire reluisant \\
\hline 19 & $\begin{array}{l}\text { 3: nu cred că este foarte colocvial, dar nici } \\
\text { informal, }\end{array}$ & $\begin{array}{l}\text { 3: je ne pense pas qu'il soit très familier, ni } \\
\text { informel }\end{array}$ \\
\hline 20 & $\begin{array}{l}\text { 3: cred că e undeva între, ca registru, că nu } \\
\text { zice }\end{array}$ & $\begin{array}{l}3 \text { : je pense qu'il est quelque part entre, } \\
\text { comme registre, qu'il ne dit pas }\end{array}$ \\
\hline \multirow[t]{2}{*}{21} & 3: elegant, ştii? zice sleek şi moderne, şi... & $\begin{array}{l}\text { 3: élégant, tu comprends? Il dit sleek et } \\
\text { moderne, et ... }\end{array}$ \\
\hline & 3: nu asta, înțelegeți? şi elegante, şi & 3: pas ça, vous comprenez? et élégantes, et \\
\hline 22 & 1: confortabile & $1:$ confortables \\
\hline 23 & $\begin{array}{l}\text { 3: da, deci mai multe şi scumpe, evident şi } \\
\text { ne-ar }\end{array}$ & $\begin{array}{l}\text { 3: oui, donc beaucoup plus et cher, } \\
\text { évidemment il nous }\end{array}$ \\
\hline
\end{tabular}




\begin{tabular}{|c|c|c|}
\hline 24 & $\begin{array}{l}\text { 3: trebui un cuvânt care să le recupereze pe } \\
\text { toate ... nu știu care }\end{array}$ & $\begin{array}{l}\text { 3: faut un mot qui récupère tout ...: je ne } \\
\text { sais lequel }\end{array}$ \\
\hline \multirow[t]{2}{*}{$25 / 26$} & 2: extravagante & 2: extravagantes? \\
\hline & $1: \mathrm{nu}$ & $1:$ non \\
\hline \multirow[t]{2}{*}{26} & $3: . .$. e deja e prea mult & 3: c'est déjà trop3: c'est déjà trop \\
\hline & $\begin{array}{l}\text { 1: prea mult şi nu recuperează ideea asta de } \\
\text { confort }\end{array}$ & $\begin{array}{l}\text { 1: trop et ne récupère pas cette idée de } \\
\text { confort }\end{array}$ \\
\hline \multirow[t]{2}{*}{27} & $1:$ extravagant nu e tocmai confortabil & $\begin{array}{l}\text { 1: extravagant n'est pas tout-à-fait } \\
\text { confortable }\end{array}$ \\
\hline & $2: \mathrm{da}$ & $2:$ oui \\
\hline $28 / 29$ & $\begin{array}{l}\text { 3: dacă zicem văcsuite? Se zice în română } \\
\text { văcsuite? ... ați auzit? }\end{array}$ & $\begin{array}{l}\text { 3: et si on disait „văcsuite” [laquées] ? Ça se } \\
\text { dit en roumain „văcsuite”?... tu l'as } \\
\text { entendu? }\end{array}$ \\
\hline 29 & $\begin{array}{l}\text { 1: se zice, da eu am auzit, dar mă duce cu } \\
\text { gândul }\end{array}$ & $\begin{array}{l}1: \text { ça se dit oui, je l'ai entendu, et ça me fait } \\
\text { penser }\end{array}$ \\
\hline \multirow[t]{2}{*}{30} & $1:$ la ceva uns, lucios & $\begin{array}{l}1: \text { à quelque chose d'oint, d'huilé, de } \\
\text { reluisant }\end{array}$ \\
\hline & 3: păi da, în sensul că sunt lucioase & 3: oui, au sens qu'elles sont reluisantes \\
\hline 31 & 3: si ultraspălate şi ultraaranjate & 3: et ultralavées et ultra-arrangées \\
\hline 32 & 1: dar văcsuit, nu ştiu, & 1: mais „văcsuit”, je ne sais pas \\
\hline 33 & $\begin{array}{l}\text { 1: are o conotație ușor negativău nu-mi } \\
\text { amintesc decât din texte de-astea, cazone, } \\
\text { de la ăia cu }\end{array}$ & $\begin{array}{l}\text { 1: cela a une connotation légèrement } \\
\text { négative, je ne me souviens que du } \\
\text { contexte de la caserne, celui avec les }\end{array}$ \\
\hline \multirow[t]{2}{*}{34} & 1: ghetele văcsuite & $1:$ bottes cirées \\
\hline & 3: problema e că trebuie să foloseşti & 3: le problème est qu'il faut utiliser \\
\hline 35 & $\begin{array}{l}\text { 3: un cuvânt care să exprime cât de cât ceva } \\
\text { adică, }\end{array}$ & 3: un mot qui exprime un peu de tout, donc \\
\hline \multirow[t]{2}{*}{36} & 3: dacă zicem elegante & 3: si nous disons élégante \\
\hline & 1: da, sau moderne, într-adevăr & 1: oui, ou bien moderne, c'est effectivement \\
\hline \multirow[t]{2}{*}{37} & 1: e prea sec & $1:$ trop sec \\
\hline & $\begin{array}{l}\text { 3: ştii, parcă, da, exact, e prea sec, spune } \\
\text { prea }\end{array}$ & $\begin{array}{l}\text { 3: tu comprends, il semble, oui, exactement, } \\
\text { il est trop sec }\end{array}$ \\
\hline 38 & 3: puțin față de cuvântul englezesc nu sunt & $\begin{array}{l}\text { 3: il dit trop peu par rapport au mot anglais } \\
\text { je n'en suis }\end{array}$ \\
\hline 39 & $\begin{array}{l}\text { 3: convinsă s-ar putea ca sleek asta să fie } \\
\text { chiar americanism, dar nu sunt sigur }\end{array}$ & $\begin{array}{l}\text { 3: pas convaincue mais u'il se pourrait que } \\
\text { ce sleek soit même un américa- nisme, } \\
\text { mais je n'en suis pas sure }\end{array}$ \\
\hline 40 & 1: da, aşa sună & $1:$ oui, ça sonne comme ça \\
\hline \multirow[t]{3}{*}{41} & 3: ca scoase din cutie? & 3: comme sorti de la boîte à cadeaux? \\
\hline & $1:$ prea multe & $1:$ c'est trop \\
\hline & $4:$ sic & $4:$ sic \\
\hline 42 & $\begin{array}{l}\text { 1: merge aşa, ca nuanță, cred că recuperează } \\
\text { multe chestii }\end{array}$ & $\begin{array}{l}\text { 1:, mais ça va comme ça comme nuance, je } \\
\text { crois que ça récupère beaucoup de choses }\end{array}$ \\
\hline 43 & $\begin{array}{l}\text { 3: dar şi pierde altele? adică nu poți să zici } \\
\text { maşini }\end{array}$ & $\begin{array}{l}\text { 3: mais ça en perd d'autres? Alors, est-ce } \\
\text { qu'on ne pourrait pas dire des voitures }\end{array}$ \\
\hline \multirow[t]{2}{*}{$44 / 45$} & $3:$ şmechere, nu? & $3:$ des voitures pour flamber, non? \\
\hline & $\begin{array}{l}\text { 1: nu, în nici un caz, că e prea } \\
3: \text { maşină nu e şmecheră? }\end{array}$ & $\begin{array}{l}\text { 1: non, en aucun cas, } \\
3: \text { une voiture ne peut pas être pour } \\
\text { flamber? }\end{array}$ \\
\hline 45 & $\begin{array}{l}\text { 1: colocvial da, dar nu poți să spui apoi şi } \\
\text { noi, şi }\end{array}$ & $\begin{array}{l}\text { 1: c'est trop familier et tu ne peux pas dire } \\
\text { après et neuves et }\end{array}$ \\
\hline \multirow[t]{2}{*}{46} & $1:$ ca scoase din cutie deci, probabil, pentru & $\begin{array}{l}\text { 1: comme sorties de la boîte à cadeaux, } \\
\text { probablement }\end{array}$ \\
\hline & 3: da, exact & 3: oui, exactement \\
\hline
\end{tabular}




\begin{tabular}{|c|c|c|}
\hline \multirow[t]{2}{*}{$47 / 49$} & 1: ambele să folosim & $1:$ il faudra utiliser les deux \\
\hline & $\begin{array}{l}\text { 2: da, dar ca scoase din cutie nu înseamnă } \\
\text { neapărat că sunt noi, nu? }\end{array}$ & $\begin{array}{l}\text { 2: oui, mais comme sorti de la boîte à } \\
\text { cadeaux ne veut pas forcément dire } \\
\text { qu'elles sont neuves, non? }\end{array}$ \\
\hline 48 & $1:$ mie asta-mi & $1:$ à moi ça \\
\hline \multirow[t]{2}{*}{49} & 1: spune, că sunt noi-nouțe scos din cutie e & $\begin{array}{l}\text { 1: me dit qu'elles sont flambant neuves } \\
\text { sorties de la boîte à cadeaux }\end{array}$ \\
\hline & 3: şi mie & $3:$ à moi aussi \\
\hline \multirow[t]{2}{*}{50} & 1: ireproşabile & 1: irréprochables \\
\hline & $1:$ scos din cutie e ca la cadou dacă l-ai scos & $\begin{array}{l}\text { 3: sorties de la boîte à cadeaux c'est comme } \\
\text { le cadeau, si tu l'as sorti }\end{array}$ \\
\hline 51 & $\begin{array}{l}\text { 1: din cutie, teoretic, trebuie sa fie nou, } \\
\text { cadoul }\end{array}$ & $\begin{array}{l}\text { 1: de la boîte, théoriquement, il doit être } \\
\text { neuf, le cadeau. }\end{array}$ \\
\hline 52 & 3: dacă aveți voi altă propunere? & 3: si vous, vous avez une autre psition? \\
\hline \multirow[t]{2}{*}{53} & $\begin{array}{l}\text { 1: nu deci chiar nu văd un singur cuvânt } \\
\text { care sa redea tot }\end{array}$ & $\begin{array}{l}1: \text { non, je ne vois même pas un seul mot } \\
\text { qui rende tout }\end{array}$ \\
\hline & $\begin{array}{l}\text { 3: dacă zici 3: ultramoderne? Vi se pare că-i } \\
\text { mai bun? }\end{array}$ & $\begin{array}{l}\text { 3: et si tu dis ultramodernes? Ça vous paraît } \\
\text { meilleur? }\end{array}$ \\
\hline $54 / 55$ & $\begin{array}{l}1: \text { şi-asta are o nuanță de extravaganță, } \\
\text { totuşi ultramodern }\end{array}$ & $\begin{array}{l}\text { 1: mais ça a une nuance d'extravagance, } \\
\text { cependant, ultramoderne, }\end{array}$ \\
\hline 56 & $\begin{array}{l}\text { 1: care nu e prezentă în text neapărat deci } \\
\text { cred că }\end{array}$ & $\begin{array}{l}\text { 1: qui n'est pas forcément présente dans le } \\
\text { texte, je pense donc que }\end{array}$ \\
\hline 57 & $\begin{array}{l}\text { 1: putem să ramânem la maşini ca scoase } \\
\text { din cutie }\end{array}$ & $\begin{array}{l}\text { 1: nous pouvons rester avec: des voitures } \\
\text { comme sorties de la boîte à cadeaux }\end{array}$ \\
\hline \multirow[t]{2}{*}{58} & $1:$ accelerează, şi aşa mai departe & $1:$ accélèrent, et ainsi de suite \\
\hline & 3: voi cum ziceti? & 3: vous qu'en dites-vous? \\
\hline \multirow[t]{2}{*}{59} & $3:$ vox populi & 3: vox populi \\
\hline & 4: maşini elegante e suficient să creeze & 4: des voitures élégantes suffit à créer \\
\hline \multirow[t]{2}{*}{60} & 4:atmosfera aia de lux & $4:$ cette atmosphère de luxe \\
\hline & 3: maşini elegante, zici tu? & 3: des voitures élégantes, tu dis? \\
\hline \multirow[t]{2}{*}{61} & 3: I can live with that voi ce ziceți? & $\begin{array}{l}\text { 3: I can live with that, et vous vous dites } \\
\text { quoi? }\end{array}$ \\
\hline & 1: Raluca, & $1:$ Raluca \\
\hline \multirow[t]{2}{*}{62} & 2: elegant nu înseamnă neapărat că & 2: élégant ne signifie pas forcément que \\
\hline & 3: Raluca se abține & 3: Raluca s'abstient \\
\hline \multirow[t]{2}{*}{63} & $2:$ e nou & $2:$ c'est neuf \\
\hline & 3: păi putem să zicem maşini noi şi elegante, & $\begin{array}{l}3: \text { bon, alors on peut dire des voitures } \\
\text { neuves et élégantes }\end{array}$ \\
\hline \multirow[t]{3}{*}{64} & $1:$ dacă asta e problema & $, 1:$ si c’est ça le problème \\
\hline & $2: \mathrm{da}, \mathrm{da}$ & 2: oui, oui \\
\hline & 3: dacă asta e da, ideea & 3: oui, si c'est ça, l'idée, c'est \\
\hline 65 & $\begin{array}{l}\text { 3: e să le exprimăm cumva pe amândouă } \\
\text { maşini noi }\end{array}$ & $\begin{array}{l}\text { 3: d'exprimer d'une certaine façon les deux: } \\
\text { des voitures neuves }\end{array}$ \\
\hline \multirow[t]{3}{*}{66} & 3: şi elegante speed along & 3: et élégantes speed along \\
\hline & $2: \mathrm{da}$ & $2:$ oui \\
\hline & $1:$ eu aş zice accelerează & $1:$ moi je dirais: accélèrent \\
\hline
\end{tabular}

